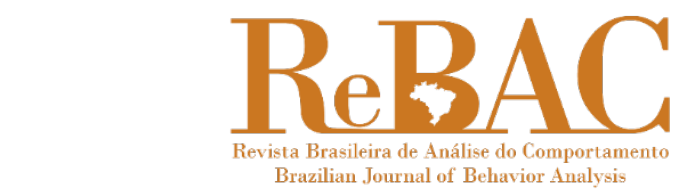

ISSN 1807-8338 - Versão Impressa | ISSN 2526-6551 - Versão Eletrônica periodicos.ufpa.br/index.php/rebac

\title{
Formação de Classes de Estímulos Equivalentes em Idosos Saudáveis: Uma Revisão
}

\author{
Stimulus Class Formation in Healthy Elderly: A Review
}

ANDRÉIA SCHMIDT ${ }^{1}$

${ }^{1}$ UNIVERSIDADE DE SÃO PAULO

\section{Resumo}

O aumento da prevalência de idosos na população requer o desenvolvimento de conhecimentos básicos sobre o declínio "cognitivo" decorrente do processo natural de envelhecimento, ou de transtornos neurocognitivos. Estudos sobre controle de estímulos e, especificamente, sobre a formação de classes de equivalência, podem ajudar a elucidar essa questão. Os objetivos desta revisão foram: a) analisar estudos sobre o desempenho de idosos saudáveis em relação à aprendizagem de relações condicionais e formação e/ou manutenção de classes de equivalência; b) descrever o desempenho de idosos nessas tarefas em comparação a participantes jovens. Após busca nas bases Periódicos Capes e PsychInfo, e busca manual em cinco periódicos específicos de Análise do Comportamento, foram selecionados para análise nove artigos que tinham como participantes idosos saudáveis, que passaram por procedimentos de ensino de relações condicionais e testes de formação de classes de equivalência. A maioria dos estudos verificou que idosos aprendem as relações condicionais ensinadas e formam classes de equivalência. Falhas em atingir critérios nos procedimentos não podem ser seguramente atribuídas à idade. Estudos que investigaram o efeito de diferentes estruturas de treino de relações condicionais apresentaram resultados inconclusivos. Os três estudos que compararam o desempenho de idosos e jovens nessas tarefas verificaram pior desempenho dos idosos. Compreender as variáveis que contribuem ou prejudicam o desempenho de idosos na aprendizagem de relações condicionais e formação de classes de equivalência requer a ampliação das pesquisas, o controle cuidadoso de características dos idosos (e.g., escolaridade), a análise dos procedimentos adotados e o aumento do número de participantes estudados.

Palavras-chave: Equivalência de estímulos, relações condicionais, estruturas de treino, controle de estímulos, idosos.

\section{Abstract}

The increase in the prevalence of elderly people in the population requires the development of basic knowledge about "cognitive" decline resulting from the natural aging process or neurocognitive disorders. Studies on stimulus control and, specifically, on equivalence class formation can help to clarify this issue. The aims of this review were: a) to analyze studies on the performance of healthy elderly people in the learning of conditional relations and the formation and / or maintenance of equivalence classes; b) describe the performance of the elderly in these tasks compared to young participants. After searching the Periódicos Capes and Psychlnfo databases, and searching manually in five specific Behavior Analysis journals, nine papers were selected for analysis, all of them with healthy elderly participants and with procedures for teaching conditional relations and equivalence class formation tests. Most studies have found that older adults learn conditional relations and form equivalence classes. Failures to meet criteria in procedures cannot be safely attributed to age. Studies that investigated the effect of different training structures have shown inconclusive results. The three studies that compared the performance of the elderly and young people in these tasks found worse performance of the elderly. Understanding the variables that contribute to or impair the elderly's performance in learning conditional relations and forming equivalence class requires more research, careful control of the elderly's characteristics (e.g., education), analysis of the procedures adopted and increasing the number of participants studied.

Keywords: Stimulus equivalence, conditional relations, training structures, stimulus control, elderly.

A autora é membro do Instituto Nacional de Ciência e Tecnologia sobre Comportamento, Cognição e Ensino (INCTECCE), financiado pelo Conselho Nacional de Pesquisa (CNPq, processo n 573972/2008-7) e Fundação de Amparo à Pesquisa do Estado de São Paulo (FAPESP, processo nº 08/57705-8).

$\triangle$ aschmidt@ffclrp.usp.br

DOI: HTTP://DX.DOI.ORG/10.18542/REBAC.V17I1.10632 
O aumento da prevalência de idosos na população requer a produção de conhecimentos básicos sobre eventual declínio "cognitivo" causado pelo processo natural de envelhecimento ou decorrente de transtornos neurocognitivos (causados ou não por demências). Estudos dessa natureza podem servir para o desenvolvimento de tecnologias de intervenção junto a essa população, tanto em nível de prevenção quanto de reabilitação. Vários campos de conhecimento, como as neurociências e a gerontologia, já identificaram essa necessidade e têm promovido avanços importantes nessa direção. A longa tradição da Análise do Comportamento no desenvolvimento de tecnologias de intervenção eficazes para diversas populações a capacita para contribuir com essa tarefa.

A despeito dessa expertise, porém, há uma quantidade proporcionalmente pequena de estudos experimentais envolvendo idosos na literatura analítico-comportamental. Em 1986, o Journal of Applied Behavior Analysis (JABA), importante periódico da área com tradição na publicação de estudos aplicados, publicou um número especial sobre gerontologia comportamental. Uma revisão publicada no JABA 24 anos depois (Trahan et al., 2011), constatou que, entre 1968 e 2010 apenas 34 artigos publicados no JABA tratavam de questões relacionadas ao envelhecimento. Até 1986, ano de publicação do número especial, foram nove artigos, sendo que dois deles envolviam algum adulto com demência. Os temas mais tratados eram o engajamento dos idosos em atividades diversas, e problemas relacionados à alimentação e incontinência urinária; a maioria dos estudos relatava pesquisas aplicadas realizadas, em sua maioria, em instituições de atendimento aos idosos. Entre 1986 e 2010, foram publicados 21 artigos (um número expressivamente maior que no período anterior), sendo que 15 deles tinham na amostra ao menos um participante com demência, o que representa uma mudança significativa, ainda que proporcionalmente esse número continuasse pequeno. Os temas dos artigos continuavam se concentrando em problemas de comportamento apresentados por idosos (e.g., comportamento agressivo e vocalizações disruptivas), talvez refletindo uma situação cultural de aumento no número de idosos com demência que necessitavam residir ou frequentar instituições e que demandavam cuidados profissionais intensivos. Até aquele momento não havia registros, naquele periódico, de pesquisas que tratassem de intervenções sobre aspectos do "funcionamento cognitivo" dos idosos.

Uma pesquisa não sistemática realizada por esta autora no JABA entre os anos de 2011 e 2018 localizou sete estudos com participantes idosos, seis deles envolvendo somente ou também idosos com demência. Como nos demais períodos pesquisados por Trahan et al. (2011), os estudos foram conduzidos em instituiç̃̃es de atendimento a idosos e os temas se mantiveram os mesmos, com o destaque para um artigo (Trahan et al., 2014) que testava uma intervenção com comunicação alternativa, tratando, portanto, de uma questão relacionada à linguagem. Desta forma, até 2018 foram publicados 41 artigos envolvendo idosos no JABA.

Seguindo nessa revisão informal, esta autora consultou outros periódicos prestigiados na área, desde a sua fundação até 2019: o Journal of The Experimental Analysis of Behavior (JEAB), The Psychological Record (TPR), a brasileira Revista Brasileira de Análise do Comportamento (REBAC) e a latina Acta Comportamentalia (AC). Em todos esses periódicos um número ainda menor de artigos envolvendo idosos foi identificado: respectivamente seis, cinco, quatro e dois. No total (incluindo o JABA), foram identificados 58 artigos publicados nesses periódicos e que tinham idosos como participantes de pesquisa, desde a fundação das revistas. Esse número é muito reduzido, especialmente se se considerar que o periódico internacional mais jovem (REBAC) tem 15 anos de existência, enquanto que os mais antigos (TPR, JEAB e JABA) têm, respectivamente, 84, 71 e 53 anos de circulação (a AC tem 28 anos de circulação). É claro que vários estudos produzidos por analistas do comportamento e que tinham idosos como participantes foram publicados em outros periódicos, inclusive revistas cujo foco é voltado especificamente para questões relacionadas ao envelhecimento, como será visto adiante nesta revisão. No entanto, não se pode ignorar que os números aqui apresentados mostram a pouca atenção que analistas do comportamento têm dado para estudos relacionados ao envelhecimento.

Essa conclusão é corroborada por outras pesquisas. Um levantamento bibliográfico sistemático realizado por Domeninconi et al. (2010) recuperou, até o ano de 2009, 32 artigos de orientação analítico-comportamental cujos participantes eram idosos, sendo que cinco deles foram publicados no número especial do JABA sobre gerontologia comportamental. Da mesma forma, Aggio et al. (2018), em uma revisão sobre artigos que relatavam intervenções analíticocomportamentais com idosos com demência, com foco específico em cognição e linguagem, localizou apenas 16 artigos. Também Goyos et al. (2009), em levantamento sobre intervenções analítico-comportamentais com idosos sobre memória, comportamento de vagar, incontinência urinária e fala desconexa, recuperou 16 estudos. Todas essas revisões, portanto, confirmam o número reduzido de estudos sobre questões relativas ao envelhecimento conduzidas por analistas do comportamento.

Especificamente em relação ao declínio decorrente do processo natural de envelhecimento, defende-se que os estudos sobre controle de estímulos podem contribuir de forma robusta para a compreensão de dificuldades chamadas "cognitivas". Se, por um lado, as neurociências têm se concentrado fortemente em estudar de que maneira as alterações 
neuroquímicas e neurofisiológicas impactam o funcionamento cognitivo do indivíduo, a Análise do Comportamento, por sua vez, procura entender funcionalmente as relações entre variáveis comportamentais e ambientais, eventualmente utilizando medidas fisiológicas para a melhor compreensão dos desempenhos discriminativos dos indivíduos. Como afirmam Steingrimsdottir e Arntzen (2014), o estudo da memória ou da atenção, por exemplo, a partir de um referencial analíticocomportamental, tem como unidade de análise as relações funcionais entre o ambiente e o comportamento. Estudar os parâmetros em que essas variáveis podem produzir (ou não) déficits de controle de estímulos poderia servir, por exemplo, para rastrear casos de demência leve ou moderada (Steingrimsdottir \& Arntzen, 2011).

Nesse sentido, muitos dos estudos de fundamentação analítico-comportamental sobre desempenhos sob controle de estímulos em idosos têm se baseado no paradigma da equivalência de estímulos (Sidman \& Tailby, 1982; Sidman,1994). Esse paradigma sugere que, a partir da aprendizagem de um conjunto de relações condicionais entre estímulos, uma série de outras relações não diretamente ensinadas pode emergir (de Rose \& Bortoloti, 2007; Sidman \& Tailby, 1982). Por exemplo, aprendemos a relacionar a palavra "bola" ao brinquedo redondo utilizado em jogos diversos, da mesma forma que podemos aprender a relacionar essa palavra a desenhos ou fotos desse objeto e a palavras de outros idiomas, como "pelota" ou "ball". A partir dessa aprendizagem, sem que haja ensino direto, podemos relacionar o objeto bola às palavras de outros idiomas, ou relacionar o objeto às suas respectivas fotos e figuras. A formação de uma classe de estímulos equivalentes é inferida quando ocorre essa extensão das relações entre estímulos que não foram diretamente relacionados. Essa extensão é descrita pelas propriedades de reflexividade, simetria e transitividade (para maior detalhamento dessas propriedades, ver de Rose, 1993 e Sidman \& Tailby, 1982). Além disso, os estímulos que compõem a classe de equivalência passam a compartilhar funções e significados, o que tem sido caracterizado como comportamento simbólico (de Rose \& Bortoloti, 2007).

A relação entre os estímulos que compõem a classe é arbitrária (ou seja, não ocorre em função de similaridade física entre eles) e é estabelecida por meio de discriminações condicionais. A tarefa mais comumente empregada para o estabelecimento dessas relações arbitrárias é a tarefa de emparelhamento ao modelo (ou, em inglês, matching to sampleMTS). Nela, o indivíduo aprende a relacionar um entre dois ou mais estímulos disponíveis (chamados de estímulos de comparação) a um estímulo modelo - como nas relações estabelecidas entre palavras da língua e seus referentes. Imaginese como exemplo a aprendizagem da relação entre a palavra bola e o brinquedo correspondente: frequentemente a criança se encontra entre alguns brinquedos/objetos e o adulto diz "Onde está a bola?" (estímulo-modelo); respostas da criança em direção à bola, e não a outros brinquedos, são seguidas por consequências reforçadoras positivas dispensadas pelo adulto, o que estabelece, ao longo de várias tentativas, a relação palavra-objeto.

As tarefas utilizadas no ensino de relações condicionais e em testes de formação de classes de equivalência podem ser consideradas como "tarefas cognitivas". Wilson e Milan (1995) argumentam que essas tarefas podem ser vistas como tarefas de resolução de problemas que demandam a formulação de regras do tipo "se...então...". Isso seria correto em dois níveis. Em um nível mais simples, na aprendizagem das discriminações condicionais, por meio das tarefas de MTS: a relação entre os estímulos modelo e comparação seriam estabelecidas por meio do reforço diferencial do desempenho do indivíduo. Em um nível mais complexo, segundo os mesmos autores, os testes de equivalência (simetria e transitividade) também demandariam a formulação de regras para que relações não diretamente ensinadas fossem derivadas nos testes.

Considerando essas características das tarefas envolvidas na formação de classes de equivalência, Wilson e Milan (1995) sugerem que procedimentos que testam a formação dessas classes poderiam ser utilizados para o estudo de prejuízos decorrentes de transtornos neurocognitivos. Compreender tais prejuízos, no entanto, depende de um passo anterior, que é entender em que medida idosos saudáveis (i.e., que não apresentam indicativos de transtornos neurocognitivos) podem ou não ter seus desempenhos em tarefas de discriminação condicional e na formação/manutenção de classes de equivalência afetados pelo processo natural de envelhecimento. Nessa perspectiva mais ampla, vários estudos sobre o desempenho de idosos saudáveis em tarefas de formação de classes de estímulos equivalentes passaram a ser conduzidos. Os objetivos da presente revisão foram: a) analisar estudos sobre o desempenho de idosos saudáveis em relação à aprendizagem de relações condicionais e formação e/ou manutenção de classes de equivalência; b) descrever o desempenho de idosos nessas tarefas em comparação a participantes jovens. A revisão aqui proposta também pode mostrar um panorama comparativo com a revisão publicada por Domeniconi et al. (2010), uma vez que, na época, as autoras localizaram apenas dois artigos científicos sobre procedimentos envolvendo equivalência de estímulos e idosos.

\section{Método}

Foi realizada uma busca de artigos nas bases Periódicos Capes e Psycholnfo em dezembro de 2019. As buscas foram feitas com as seguintes combinações de termos: stimulus equivalence and elderly; stimulus equivalence and old. Além disso, foi feita uma busca manual nos periódicos Journal of the Experimental Analysis of Behavior (JEAB), Journal of Applied Behavior Analysis (JABA), The Psychological Record (TPR), Acta Comportamentalia (AC) e Revista Brasileira de Análise do 
Comportamento (REBAC), com as mesmas combinações de termos. Os critérios de seleção dos artigos foram: ser um artigo empírico; ter como participantes idosos, sem indicativos ou diagnóstico de transtorno neurocognitivo (portanto, saudáveis); e descrever procedimento de ensino de relações condicionais e de formação de classes de equivalência. Foram critérios de exclusão: ser artigo teórico ou de revisão; ter como participantes animais não humanos, crianças, jovens, adultos com menos de 60 anos ou somente participantes idosos com transtorno neurocognitivo; descrever apenas procedimentos de ensino de relações condicionais, sem a condução de testes de formação de classes de equivalência.

Na busca inicial foram encontrados 42 artigos na base Periódicos Capes; 117 na base Psychlnfo; nove artigos no periódico JEAB; sete no periódico JABA; 27 no TPR; dois na AC e quatro na REBAC. A busca inicial localizou, portanto, 208 artigos. Depois de excluídas as entradas idênticas, permaneceram para análise 203 artigos. Em seguida, foram lidos os títulos de todas as pesquisas, para uma primeira identificação e exclusão dos artigos que não atendiam aos critérios de inclusão. Após essa etapa, restaram 48 artigos para análise dos resumos. A partir da leitura dos resumos, chegou-se ao número final de nove artigos para análise, publicados entre 1995 e 2015. Dois artigos incluíam participantes com idade entre 55 e 59 anos. No artigo de Saunders et al. (2005) havia um participante de 56 anos (entre $12 \mathrm{com} 60$ ou mais) e no artigo de Gallagher e Keenan (2009) é reportada a presença de um participante de 57 anos no Experimento 2. Apesar disso, ambos os artigos foram incluídos na análise por esses participantes representarem uma porcentagem muito pequena da amostra estudada. A seleção dos artigos foi realizada apenas pela autora deste artigo.

Em função do número reduzido de artigos, optou-se por fazer uma análise individual dos estudos, ressaltando os principais resultados encontrados e discutindo-os.

\section{Resultados e Discussão}

Os artigos revisados foram publicados em diferentes periódicos: European Journal of Behavior Analysis (1), Journal of Gerontology: Psychological Sciences (01), Psicothema(1), The Psychological Record(2), Acta Comportamentalia(1), Revista Argentina de Ciências del Comportamiento (1), Psicologia Argumento(1), e Revista Brasileira de Análise do Comportamento (1). Observa-se, portanto, que cinco artigos foram publicados em revistas específicas de análise do comportamento e, os demais, em revistas de conteúdo mais amplo.

Os estudos que investigam o desempenho de idosos saudáveis em tarefas que envolvem a aprendizagem de relações condicionais e a emergência de relações derivadas - simetria e transitividade (formação de classes de estímulos equivalentes), utilizam procedimentos muito similares. A tarefa básica utilizada é a de MTS, tal como em estudos com populações diversas (de Rose, 1993; Sidman, 1994). Quando, tanto o estímulo-modelo, quanto os estímulos de comparação ficam disponíveis à inspeção do participante durante todo o tempo de duração da tentativa, diz-se que a tarefa é de MTS simultâneo; quando o estímulo-modelo é apresentado e, após uma resposta de observação (e.g., um toque sobre ele na tela do computador) ele é suprimido e são apresentados os estímulos de comparação após um período de tempo (que pode variar de zero a n segundos), diz-se que a tarefa é de MTS com atraso. Após a seleção de um dos estímulos de comparação pelo participante, são fornecidas consequências diferenciais para acertos e erros, no caso de tentativas de ensino, ou a apresentação de uma nova tentativa, no caso de tentativas de teste ou sonda.

Em geral, são ensinados dois ou três conjuntos de relações condicionais separadamente (e.g., AB e AC, em uma estrutura de treino um para muitos). Em seguida, são apresentados blocos incluindo tentativas reforçadas de todos os conjuntos de relações ensinadas (e.g., $A B / A C$ ), seguidos de blocos de tentativas misturadas sem consequências diferenciais (extinção). Na sequência, são apresentados testes de simetria (e.g., BA e CA) e de transitividade (BC e CB). Algumas variações desse procedimento básico podem ser encontradas, como a inserção de um bloco de tentativas em que ocorre a redução da probabilidade de tentativas reforçadas antes de se apresentar um bloco de tentativas em extinção. A estrutura de treino pode variar: na estrutura linear, os estímulos que servem como estímulos de comparação em uma fase de treino são empregados como estímulos-modelo em uma fase subsequente (e.g., AB, depois BC, CD, e assim por diante). Na estrutura um para muitos um mesmo conjunto de estímulos serve como estímulo-modelo nas diferentes fases do treino (e.g., $A B, A C, A D$, etc). Finalmente, na estrutura muitos para um, o mesmo conjunto de estímulos é relacionado a diferentes estímulos-modelo nas fases consecutivas de ensino (e.g., BA, CA, DA, etc). Independentemente dessas variações, porém, os resultados obtidos nos estudos podem ser comparados entre si. A Tabela 1 apresenta os estudos realizados com idosos saudáveis sobre aprendizagem de relações condicionais e formação de classes de equivalência. 
Tabela 1.

Pesquisas Sobre Equivalência de Estímulos com Idosos e Jovens Saudáveis

\begin{tabular}{|c|c|c|c|c|c|c|c|c|c|c|c|c|c|}
\hline \multirow[b]{2}{*}{ Artigo } & \multirow[b]{2}{*}{$\mathrm{N}$} & \multirow[b]{2}{*}{ Idade } & \multirow[b]{2}{*}{$\begin{array}{l}\text { Anos } \\
\text { estudo }\end{array}$} & \multirow[b]{2}{*}{$\begin{array}{l}\text { Tipo } \\
\text { treino }\end{array}$} & \multicolumn{3}{|c|}{ Idosos } & \multirow[b]{2}{*}{$\mathrm{N}$} & \multicolumn{3}{|c|}{ Jovens } & \multirow[b]{2}{*}{$\begin{array}{l}\% \\
\text { Equiv. } \\
\star\end{array}$} & \multirow[b]{2}{*}{ Principais conclusões } \\
\hline & & & & & $\begin{array}{l}\text { Estr. } \\
\text { treino }\end{array}$ & $\begin{array}{l}\% \\
\text { Aprend* }\end{array}$ & $\begin{array}{l}\% \\
\text { Equiv* }\end{array}$ & & Idade & $\begin{array}{l}\text { Anos } \\
\text { estudo }\end{array}$ & $\begin{array}{l}\% \\
\text { Apre } \\
\text { nd }^{*}\end{array}$ & & \\
\hline $\begin{array}{l}\text { Wilson \& Milan } \\
\qquad(1995)\end{array}$ & 20 & $62-81$ & $\begin{array}{l}17,5 \\
\text { (méd) }\end{array}$ & Sim & LS & $\mathrm{NI}$ & 45 & 20 & $19-22$ & $\begin{array}{l}14,5 \\
\text { (méd) }\end{array}$ & $\mathrm{NI}$ & 80 & $\begin{array}{l}\text { Idosos têm mais dificuldades que jovens } \\
\text { para aprender relações condicionais } \\
\text { formar classes; suas latências de resposta } \\
\text { são mais longas }\end{array}$ \\
\hline $\begin{array}{l}\text { Pérez-Gonzales } \\
\text { \& Moreno- } \\
\text { Sierra (1999) }\end{array}$ & 6 & $66-74$ & $\mathrm{NI}$ & Sim & LS & 75 & 100 & 4 & $13-53$ & $\mathrm{NI}$ & 100 & 100 & $\begin{array}{l}\text { Idosos têm mais dificuldades que jovens } \\
\text { para aprender relações condicionais }\end{array}$ \\
\hline $\begin{array}{l}\text { Cáceres- } \\
\text { Pachón et al. } \\
\text { (2015) }\end{array}$ & 4 & $70-73$ & Mín. 4 & Sim & LS & 100 & $18,7^{\star \star}$ & 8 & $17-20$ & EM-Sup & 100 & $53^{\star \star}$ & $\begin{array}{l}\text { Idosos têm mais dificuldades que jovens } \\
\text { para aprender relações condicionais } \\
\text { formar classes. Procedimentos de } \\
\text { consequências específicas não têm efeitos } \\
\text { diferenciais sobre a aprendizagem }\end{array}$ \\
\hline $\begin{array}{l}\text { Saunders et al. } \\
\qquad(2005)\end{array}$ & 12 & $56-89$ & $8-18$ & Sim & $\begin{array}{l}\text { OTM } \\
\text { MTO } \\
\text { LS }\end{array}$ & $\begin{array}{l}83 \\
91 \\
66\end{array}$ & $\begin{array}{l}30-90 \text { em } \\
\text { todas } \\
\text { condições }\end{array}$ & - & - & - & - & - & $\begin{array}{l}\text { A formação de classes não se relacionou ao } \\
\text { número de comparações no treino e nem à } \\
\text { estrutura de treino. Nenhum idoso formou } \\
\text { todas as classes }\end{array}$ \\
\hline $\begin{array}{c}\text { Gallagher \& } \\
\text { Keenan (2009) }\end{array}$ & 39 & $57-95$ & $\mathrm{NI}$ & Sim & LS & 92 & 74 & - & - & - & - & - & $\begin{array}{l}\text { A formação de equivalência parece ter } \\
\text { relação com o resultado no MEEM (>27) }\end{array}$ \\
\hline $\begin{array}{c}\text { Haydu \& } \\
\text { Moraes (2009) }\end{array}$ & 18 & 61 (méd) & $\mathrm{NI}$ & Sim & LS & $\begin{array}{l}100 \text { (6ss) } \\
100 \text { (4ss) }\end{array}$ & $\begin{array}{l}83 \text { (6ss) } \\
88 \text { (4ss) }\end{array}$ & - & - & - & - & - & $\begin{array}{l}\text { Sem diferenças em relação ao número de } \\
\text { estímulos das classes }\end{array}$ \\
\hline $\begin{array}{l}\text { Aggio et al. } \\
(2008)\end{array}$ & 3 & $76-83$ & $4-17$ & Sim & MTO & 100 & 100 & - & - & - & - & - & $\begin{array}{l}\text { Todos formaram classes de equivalência, } \\
\text { possivelmente em função da estrutura de } \\
\text { treino e do ensino "simples para o } \\
\text { complexo" }\end{array}$ \\
\hline
\end{tabular}




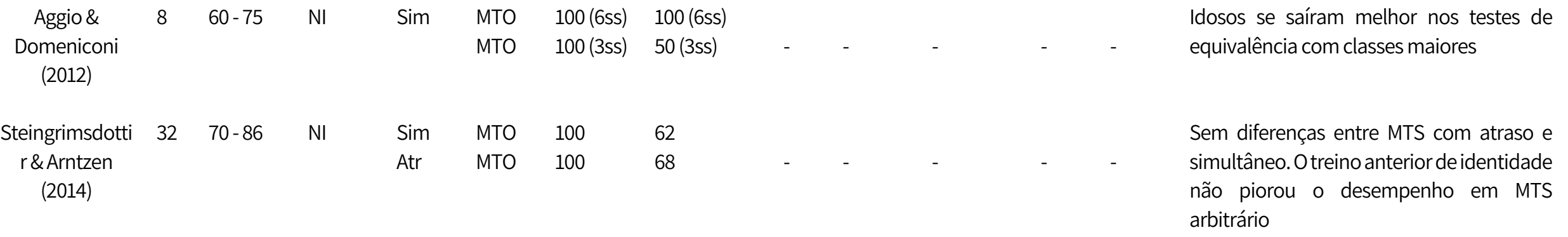

Nota: N - número de participantes - no estudo de Gallagher e Keenan (2009) foram incluídos aqui apenas os participantes que não apresentavam indicativos de comprometimento cognitivo; NI - não informado; Estrut = estrutura; ss - estímulos; Sim - MTS simultâneo; Atr - MTS com atraso; OTM - estrutura um para muitos; MTO estrutura muitos para um; LS - estrutura linear; * - porcentagem de participantes que atingiram critério de desempenho na aprendizagem de relações condicionais (Aprend) e nos testes de equivalência (Equiv); ${ }^{\star \star}$ - porcentagem de testes de simetria e transitividade completados dentro do critério estabelecido. 
Comparações entre o desempenho de idosos e de participantes jovens na aprendizagem de relações condicionais e formação de classes de equivalência. Wilson e Milan (1995) publicaram o primeiro estudo comparando o desempenho de idosos saudáveis ( $n=20$, sem indicativos de problemas cognitivos) e universitários $(n=20)$ na aprendizagem de relações condicionais visuais-visuais ( $A B$ e $A C$ ) e em testes combinados de equivalência (BC e CB). Os estímulos empregados foram letras gregas. Os dois grupos foram pareados em relação ao número de anos de estudo e em relação ao desempenho no Mini Exame de Estado Mental (MEEM - Folstein et al., 1975), que é um teste de rastreamento cognitivo. Os autores não relatam o desempenho dos participantes na aprendizagem das relações condicionais, mas apresentam os resultados dos grupos em relação à formação de classes de equivalência (testes de transitividade) e latência de respostas. Um número significativamente maior de universitários apresentou formação de classes de equivalência (16 em 20) em comparação aos idosos (nove em 20). No entanto, não foram encontradas diferenças, em termos de número de tentativas para atingir o critério, entre idosos e jovens que apresentaram formação de classes (portanto, variabilidade quanto ao desempenho dentro do grupo, mas desempenhos similares dos participantes de ambos os grupos que atingiram os critérios propostos). Os dados de latência foram comparados apenas entre os indivíduos que demonstraram equivalência, e as respostas dos idosos foram significativamente mais lentas que as dos jovens. Os autores sugeriram que as dificuldades encontradas por idosos na formação de classes de equivalência podem ser um indicador precoce de prejuízos causados pela idade na aprendizagem e na cognição, ainda que em indivíduos saudáveis.

Estudos posteriores nessa mesma linha chegaram a resultados similares. Pérez-Gonzáles e Moreno-Sierra (1999) ensinaram a uma adolescente (13 anos), três adultos (21, 44 e 53 anos) e quatro idosos (66, 67, 70 e 74 anos) relações condicionais entre estímulos visuais ( $A B$ e $B C$ ) e testaram a emergência de relações de simetria (BA e $C B$ ) e de transitividade/equivalência ( $A C$ e $C A$ ). Os estímulos eram figuras geométricas (triângulo, retângulo e círculo) e formas simples (uma cruz e uma estrela). Os quatro participantes idosos precisaram de um número maior de tentativas de treino para atingir os critérios de aprendizagem das relações $A B$ e $B C$, em comparação aos participantes mais jovens. Um dos idosos precisou de procedimentos remediativos de ensino para concluir a fase de ensino de relações $A B / B C$. Todos os participantes apresentaram emergência de simetria e transitividade, indicando formação de classes de equivalência, inclusive dois idosos adicionais (além dos quatro anteriormente testados) que passaram por um procedimento modificado de treino.

Outro estudo que comparou o desempenho de jovens e idosos, além de investigar um possível procedimento adicional para facilitar o desempenho de idosos em tarefas de discriminação condicional e formação de classes de equivalência, foi o de Cáceres Pachon et al. (2015). O estudo comparou o desempenho de jovens de nível médio (17 e 18 anos), universitários (19 e 20 anos) e idosos saudáveis (70 a 73 anos - quatro indivíduos em cada grupo) na aprendizagem de relações condicionais $(A B$ e $B C$ ) e na derivação de relações de simetria (BA e $C B$ ) e transitividade/equivalência ( $A C$ e CA). Os autores testaram se o procedimento de consequências específicas para relações de diferentes classes facilitaria a aprendizagem das relações condicionais. Para isso, adotaram um delineamento de linha de base múltipla entre os participantes de cada grupo, de forma que as consequências específicas eram introduzidas em diferentes fases do ensino para cada participante. As consequências específicas empregadas foram consequências verbais, fichas correspondentes a pontos e moedas (e.g., elogios para as relações envolvendo os estímulos da classe $\mathrm{A} 1 \mathrm{~B} 1 \mathrm{C} 1$; fichas para as relações da classe $\mathrm{A} 2 \mathrm{~B} 2 \mathrm{C} 2$ e moedas de cêntimos de euro para as relações da classe A3B3C3).

Além de replicar resultados anteriores sobre a comparação de desempenho entre jovens e idosos (os jovens aprenderam mais rapidamente as relações condicionais ensinadas e apresentaram maior número de relações emergentes de simetria e transitividade, em comparação aos idosos), o estudo de Cáceres Pachon et al. (2015) concluiu que a aplicação do procedimento de consequências específicas não apresentou qualquer efeito facilitador na aprendizagem das relações condicionais em nenhum dos grupos estudados.

Esses três estudos (Cáceres Pachon et al., 2015; Pérez-Gonzáles \& Moreno-Sierra, 1999; Wilson \& Milan, 1995) atestam diferenças de desempenho entre jovens e idosos saudáveis, tanto na aprendizagem de relações condicionais, quanto na emergência de relações não diretamente treinadas (simetria e transitividade, que atestam a formação de classes de equivalência). A principal diferença refere-se à quantidade de treino necessária para a aprendizagem, o que significa que idosos necessitaram de treinos mais extensos para atingir os critérios de aprendizagem estabelecidos, em comparação aos jovens. Um dos estudos (Wilson \& Milan, 1995) também verificou diferença na latência das respostas, ou seja, os idosos necessitaram de um tempo maior para realizar as escolhas nas tarefas. Apesar de os resultados obtidos serem semelhantes, é preciso cautela na sua interpretação. Os resultados obtidos por Pérez-Gonzáles e Moreno-Sierra (1999), assim como os de Cáceres Pachon et al. (2015) foram obtidos a partir de amostras pequenas, com 
grande variabilidade de idade e cujas informações sobre escolaridade e nível de funcionamento cognitivo são desconhecidas. Apesar de serem estudos com limitações importantes a serem ponderadas, também são estudos interessantes na medida em que revelam diferenças regulares de desempenho entre jovens e idosos, que eventualmente podem estar relacionadas ao envelhecimento.

Possíveis variáveis determinantes do desempenho de idosos na formação de classes de equivalência. Seis estudos investigaram o papel de variáveis de procedimento e da condição "cognitiva" dos idosos na sua aprendizagem de relações condicionais e na formação de classes de equivalência.

A interferência de eventuais perdas cognitivas, aferidas pelo Mini Exame de Estado Mental (MEEM), na aprendizagem de relações condicionais e na formação de classes de equivalência foi investigada por Gallagher e Keenan (2009). Os autores realizaram três experimentos, nos quais participaram, no total, 45 indivíduos com idades entre 57 e 95 anos. Para a presente análise, no entanto, foram considerados os resultados de 39 idosos, que pontuaram acima da nota de corte no MEEM (24 pontos) - portanto, indivíduos sem indicativos de declínio cognitivo. Foram ensinados dois conjuntos de relações condicionais ( $A B$ e $B C$ ), que permitiam a formação de duas classes com três estímulos visuais cada (duas sílabas sem sentido, duas formas geométricas e dois sinais gráficos - @ e \&). Foram testadas a simetria e a transitividade. Os autores defenderam que a formação de equivalência estava fortemente relacionada ao escore obtido no MEEM: 27 dos 30 idosos com escore de 27 ou mais no MEEM atingiram os critérios estabelecidos para formação de classes de equivalência, o que levou os autores a concluir que essa seria a pontuação mínima necessária para que os indivíduos formassem classes de equivalência. Outros estudos, porém, mostraram que mesmo idosos com pontuação menor que 27 (ou mesmo menor que 24) no MEEM podem apresentar desempenho compatível com a formação de classes, desde que arranjadas condições favoráveis de treino das relações condicionais (e.g., Aggio et al., 2008; Ducatti \& Schmidt, 2016), o que coloca em questão as conclusões gerais de Gallagher e Keenan (2009). Talvez a indicação de maior interesse do estudo desses autores seja a ideia de que as tarefas de aprendizagem de relações condicionais e de formação de classes podem eventualmente servir como uma espécie de rastreamento para eventuais problemas cognitivos, ideia ainda não testada de forma sistemática por analistas do comportamento.

Outro conjunto de estudos investigou aspectos estruturais do procedimento de ensino de relações condicionais que poderiam (ou não) favorecer a emergência de formação de classes de equivalência entre idosos. Saunders et al. (2005) testaram a possível interferência da estrutura de treino entre os conjuntos de estímulo (linear, muitos para um e um para muitos), o número de classes formadas em cada treino (duas, três ou quatro) e o tamanho de cada classe (três ou quatro estímulos) sobre a aprendizagem de relações condicionais e a emergência de classes de equivalência. Participaram 12 pessoas com idades entre 56 e 89 anos e com escolaridade entre oito e 18 anos. Em cada estrutura de treino os participantes aprenderam seis conjuntos de relações condicionais. Em cada conjunto de treino, o número de estímulos de comparação por tentativa era correspondente ao número de classes que se esperava serem formadas a partir do treino (duas, três ou quatro). É preciso destacar que, quanto maior o número de classes a serem formadas, maior o número de tentativas de treino, e maior a exposição dos participantes à tarefa. Após a aprendizagem de cada conjunto, os participantes eram expostos a testes de simetria, e de transitividade e/ou transitividade simétrica, a depender da estrutura de treino em vigor. Apesar da variabilidade do desempenho dos participantes, não se verificou diferença na aprendizagem das relações condicionais na comparação entre as diferentes estruturas de treino. Observou-se, porém, que quanto maior a quantidade de classes e maior o número de estímulos de comparação, maior o número de tentativas de treino necessárias para que os participantes alcançassem o critério de aprendizagem.

Nenhum participante do Experimento 1 de Saunders et al. (2005) apresentou emergência de equivalência para todos os conjuntos de treino, porém todos mostraram evidência de formação de classes para, no mínimo, cinco dos 18 conjuntos. A formação de classes não se mostrou mais provável em função do número de estímulos de comparação no treino e nem como função da estrutura de treino. Outros seis indivíduos (idades entre 58 e 75 anos) participaram de um segundo experimento, que replicava o primeiro, mas que usava tarefas de MTS com atraso de zero segundos. A partir dessa modificação, verificou-se diminuição no número de tentativas de treino para que os critérios de aprendizagem fossem atingidos, em todas as configurações (dois, três ou quatro comparações) e estruturas de treino. Além disso, verificou-se maior número de classes de equivalência formadas em comparação ao Experimento 1, com clara vantagem para as estruturas um para muitos e muitos para um, em comparação à linear. Apesar do objetivo central desse estudo não ser a comparação do desempenho dos idosos com um grupo de indivíduos mais jovens, Saunders et al. (2005) procederem a uma comparação dos resultados de seus participantes com os de universitários participantes dos estudos de Arntzen e Holth $(1997,2000)$ e Holth e Arntzen (2000), que adotavam procedimentos semelhantes. Nessa comparação, os idosos apresentaram desempenhos piores na aprendizagem das relações condicionais e nos testes em 
procedimentos que adotavam a estrutura de treino muitos para um, mas desempenhos similares (e até melhores) em outras estruturas de treino. Essa comparação, porém, deve ser vista com muita cautela em função das muitas diferenças entre os experimentos.

O estudo de Saunders et al. (2005) é importante por sugerir parâmetros de treino que podem auxiliar a aprendizagem de relações condicionais por idosos saudáveis (apesar de não haver nenhuma informação sobre aspectos cognitivos dos participantes dos experimentos), e a formação de classes de equivalência. Sua principal limitação, no entanto, está relacionada ao estudo de um número elevado de variáveis, o que pode gerar dificuldades em separar o papel específico de cada uma no conjunto dos resultados. Além disso, a grande variabilidade no perfil dos participantes em relação à idade e à escolaridade também pode ter interferido nos resultados obtidos. Apesar de nenhum estudo posterior ter replicado de forma sistemática os parâmetros adotados por Saunders et al. (2005), outras pesquisas seguiram testando variações de procedimentos que, eventualmente, pudessem favorecer o desempenho de idosos na aprendizagem de relações condicionais arbitrárias e na derivação de relações de equivalência.

Aggio et al. (2008) testaram a estrutura de treino muitos para um no ensino de relações condicionais (BA e CA) e teste de formação de três classes de três estímulos visuais cada para três idosos. Os participantes tinham entre 76 e 83 anos, no mínimo quatro anos de escolaridade e escores no MEEM de 24 a 27 . Todos aprenderam as relações condicionais com um número variado de blocos de treino e foi observada emergência imediata de formação de classes. Além da estrutura de treino, as autoras concluem que a sequência de treino empregada (do simples para o complexo) também foi uma variável relevante para os resultados alcançados. Esse estudo não apresenta fortes evidências de que a estrutura de treino muitos para um seja superior a outras, uma vez que não houve comparações nesse sentido, mas os resultados fortalecem a ideia de que, com os procedimentos apropriados, idosos saudáveis, mesmo os de idade avançada e com escores inferiores a 27 no MEEM, formam classes de equivalência.

Steingrimsdottir e Arntzen (2014) realizaram uma pesquisa em que investigaram o desempenho de idosos saudáveis na aprendizagem de relações condicionais de identidade e arbitrárias; nessas últimas, foi comparado o desempenho dos idosos em tarefas de MTS simultâneo e com atraso e a emergência de formação de classes de equivalência. Além disso, os autores estudaram se um treino em relações de identidade teria algum efeito sobre a aprendizagem de relações arbitrárias. Participaram 32 idosos sem queixa de comprometimento cognitivo, com idade entre 70 e 86 anos e escores no MEEM entre 28 e 30. Os idosos foram divididos em quatro grupos; dois deles aprenderam relações condicionais arbitrárias (AC/BC) em tarefas de MTS simultâneo e os outros dois em tarefas de MTS com atraso de zero segundos. Os grupos de MTS simultâneo aprenderam as relações arbitrárias antes (um grupo) ou depois (outro grupo) de um treino de identidade. O mesmo ocorreu com os grupos de MTS com atraso.

Todos os participantes demonstraram desempenho de identidade e aprenderam as relações condicionais. No entanto, o grupo que aprendeu as relações arbitrárias por MTS simultâneo, antes do treino de identidade, teve maior número de participantes que desistiram da pesquisa, em comparação às demais condições. Não foram verificadas diferenças entre os grupos em relação à aprendizagem de relações condicionais e nem em relação à formação de classes de equivalência. No geral, somente $65 \%$ da amostra demonstrou formação de classes e $78 \%$ demonstrou emergência de simetria. Esse estudo, diferentemente do de Saunders et al. (2005) não demonstrou superioridade do treino com atraso para a aprendizagem de relações condicionais por idosos e nem na probabilidade de formação de classes de equivalência. Os resultados obtidos nos dois estudos, portanto, deixam dúvidas sobre se, e como, o uso de atraso nas tarefas de ensino de relações condicionais pode interferir, não apenas na aprendizagem em si, mas também na posterior formação de classes de equivalência.

Buscando testar procedimentos que pudessem facilitar a aprendizagem de relações condicionais, formação e manutenção de classes de equivalência por pessoas idosas, Haydu e Morais (2009) conduziram um estudo com 18 mulheres saudáveis, cuja média de idade era 61 anos (não foi informada a faixa etária das participantes). A variável independente testada foi o tamanho das classes. Todas as mulheres aprenderam relações condicionais visuais-visuais que permitiam a formação de seis classes de equivalência (estrutura de treino linear). Metade das participantes aprendeu, inicialmente, seis classes compostas por seis estímulos (foto, nome, profissão, hobby, um objeto e um animal) e, após os testes, outras seis classes, cada uma composta por quatro estímulos (foto, nome, profissão e hobby). A ordem foi inversa para a outra metade das participantes. Foram testadas todas as relações de simetria e todas as relações de transitividade possíveis (e.g., AC, BD e DA) para cada conjunto de classes ensinadas. Seis semanas após o encerramento de cada conjunto de ensino, os testes de equivalência foram novamente conduzidos para atestar a manutenção das classes.

Na fase de ensino, Haydu e Morais (2009) verificaram maior repetição média de blocos de treino para a aprendizagem das relações que constituíam as classes com maior número de estímulos. A maior dificuldade verificada 
para a aprendizagem das participantes ocorreu nos blocos de ensino misto, em que todas as relações eram apresentadas em conjunto. Tal dificuldade com a apresentação de várias relações no mesmo bloco (seja em tentativas reforçadas, seja em tentativas de teste) foi registrada em outros estudos (e.g., Ducatti \& Schmidt, 2016). Nos testes de formação de classes (logo após o treino), 15 das 18 participantes atingiram o critério de $90 \%$ de acertos para as classes com maior número de estímulos, e 16 das 18 tiveram o mesmo desempenho em relação às classes com maior número de estímulos. Nos testes de manutenção, 10 das 18 participantes mantiveram o desempenho em relação às classes com maior número de estímulos, e 11 das 18 o fizeram em relação às classes com menor número de estímulos.

Em geral, o estudo de Haydu e Morais (2009) não revelou diferenças na aprendizagem e manutenção de classes de equivalência em função do número de estímulos que as constituíam, mas mostrou um desempenho superior das participantes em comparação a outros estudos (Saunders et al., 2005; Wilson \& Milan, 1995). As autoras destacam que isso pode ter ocorrido em função dos procedimentos de ensino adotados, mas há outros fatores não mencionados que devem ser considerados, como a idade dos participantes, seu nível de escolaridade, condições cognitivas e natureza dos estímulos (estímulos familiares, em comparação a letras gregas ou figuras abstratas utilizadas nos demais estudos citados). Sabe-se que essas variáveis podem ser muito relevantes em tarefas de discriminação condicional e testes de formação de classes de equivalência (Fields et al., 2012). A variabilidade de resultados entre os estudos reforça a necessidade de se controlar melhor a composição de amostras de idosos em pesquisas.

Aggio e Domeniconi (2012) realizaram um estudo similar ao de Haydu e Morais (2009) com oito idosos (idades entre 60 e 75 anos). As autoras investigaram a formação e manutenção de três classes de equivalência de diferentes tamanhos (três e seis estímulos), empregando a estrutura de treino muitos para um. Metade dos participantes aprendeu relações condicionais que comporiam três classes com três estímulos cada e a outra metade, três classes com seis estímulos cada (todos visuais). Testes de simetria e de transitividade foram conduzidos para todos os participantes logo após o ensino e após seis semanas (manutenção). O ensino era gradual, com várias etapas prevendo diminuição de probabilidade de consequências diferenciais e introdução gradual de novas relações. Nesse estudo, caso os participantes não apresentassem emergência imediata das relações transitivas, eram reexpostos aos testes. Todos os participantes aprenderam as relações condicionais ensinadas de forma similar (independentemente do número de estímulos por classe) e apresentaram formação de classes de equivalência. No entanto, apesar da emergência imediata ter ocorrido para todos os participantes que aprenderam as classes maiores, apenas dois participantes que aprenderam as classes menores apresentaram emergência imediata das classes. Nenhum participante que aprendeu as classes menores apresentou a manutenção das classes após seis semanas (desempenhos entre 40 e 60\% de acertos nos testes), e todos os participantes que aprenderam as classes maiores atingiram os critérios para demonstração das classes. Diferentemente de Haydu e Morais (2009), Aggio e Domeniconi (2012) verificaram diferenças na manutenção das classes em função do seu tamanho, o que pode ser um indicativo de que essa variável possa interagir com outras, como a estrutura de treino empregada. No entanto, o número reduzido de idosos em cada condição deixa dúvidas sobre o grau de generalidade dessa conclusão.

Em conjunto, os resultados obtidos por Saunders et al. (2005), Haydu e Morais (2009) e por Aggio e Domeniconi (2012) ainda deixam dúvidas sobre o papel da estrutura de treino na aprendizagem de relações condicionais e sobre como essa variável interage com outros fatores específicos do procedimento, como o tipo de ensino adotado (gradual ou por tentativa e erro, com ou sem dicas, etc.) e o tipo de estímulo (familiar ou não). Dessa forma, questões metodológicas que podem afetar o desempenho dos idosos na aprendizagem de relações condicionais e na formação de classes de equivalência ainda demandam mais investigações.

\section{Conclusões}

Apesar de aparentemente inequívoca a relação entre idade e desempenho nas tarefas estudadas (com clara vantagem para os participantes jovens), é preciso considerar que a escolaridade dos idosos foi um fator pouco controlado nos estudos e essa pode ser uma variável interveniente nos resultados discutidos. Com exceção do estudo de Wilson e Milan (1995), que controlou a escolaridade dos grupos de idosos e jovens, todos os demais tinham amostras de jovens com escolaridade superior (por vezes muito superior) à dos idosos. Certamente a experiência escolar desempenha um papel importante no ensino de desempenhos relacionais para os indivíduos, desempenhos requeridos em tarefas de formação de classes de equivalência, por exemplo.

Ardila et al. (2000) chamam a atenção para esse fato em relação aos resultados obtidos por indivíduos escolarizados em comparação aos de menor escolaridade em testes neuropsicológicos. Para esses autores, pessoas com alto nível de escolaridade são muito estimuladas a realizar tarefas que requerem o estabelecimento de relações lógicas entre situações e estímulos (e.g., situações do tipo "se..., então..."). Assim sendo, é natural que 
esses indivíduos mais escolarizados apresentem desempenhos melhores, em comparação a indivíduos menos escolarizados, como verificado em diversos estudos e adaptações de testes que levam em conta a escolaridade do indivíduo (como o MEEM, por exemplo). O problema, então, de se constituir amostras muito heterogêneas de idosos em relação à idade é a interpretação de resultados: as diferenças eventualmente encontradas podem ser devidas a variáveis muito anteriores ao envelhecimento e não se referirem especificamente a este aspecto.

É preciso considerar, também, que em todos os estudos com idosos saudáveis vários participantes (inclusive jovens dos grupos controle) não demonstraram emergência de relações de simetria e de transitividade (equivalência), mesmo em se tratando de indivíduos escolarizados. Esse resultado deve ser analisado com muita atenção, uma vez que ele sugere que a formação de classes de estímulos equivalentes depende de condições que não foram suficientemente esclarecidas e que, provavelmente, vão além da escolarização ou do envelhecimento. Independentemente dessas questões, os resultados obtidos nos estudos aqui descritos sugerem dificuldades específicas dos idosos que merecem investigação adicional, como as dificuldades verificadas entre muitos deles em demonstrar relações simétricas e em manter o desempenho atingido com relações ensinadas individualmente, quando essas relações são apresentadas em conjunto.

Além disso, os resultados obtidos em relação ao papel de variáveis metodológicas, como a estrutura de treino e o tipo de tarefa apresentada, são inconclusivos. O desenvolvimento de intervenções que envolvam tarefas de controle de estímulos, no entanto, requer conclusões sólidas a esse respeito. Estudos como o de Ducatti e Schmidt (2006) com idosos com transtorno neurocognitivo demonstrou a dificuldade desses participantes ainda na fase de aprendizagem de relações condicionais e a necessidade de se desenvolver estratégias adicionais para favorecer a aprendizagem desses participantes.

Essa revisão tem algumas limitações. A principal refere-se ao fato de que a seleção dos artigos para análise foi feita apenas por uma autora, o que pode ter gerado algum viés não intencional, apesar do critério de escolha de artigos que incluíssem idosos sem indicativos de transtornos neurocognitivos como participantes em procedimentos envolvendo formação de classes de equivalência ser bastante claro. Essa seleção feita por uma única autora, por exemplo, fez com que dois dos artigos analisados incluíssem alguns participantes com menos de 60 anos (decisão explicada anteriormente). Apesar disso, entende-se que a revisão apresentada pode contribuir com pesquisadores interessados no estudo das relações entre envelhecimento e desempenho em tarefas relacionais.

De modo geral, observou-se que o interesse de analistas do comportamento por estudos envolvendo idosos saudáveis sobre equivalência de estímulos aumentou nos últimos anos, em comparação aos resultados obtidos por Domeniconi et al. (2010): enquanto as autoras localizaram apenas dois estudos com essa especificação até 2009 (Pérez-González \& Moreno-Sierra, 1999 e Wilson \& Milan, 1995), o presente artigo localizou sete artigos adicionais, todos publicados após 2010. Em conjunto, as pesquisas aqui discutidas indicam que 0 estudo do desempenho de idosos saudáveis na formação de classes de estímulos equivalentes necessita de ampliação, sobretudo se se considerar ser esse um caminho possível para o desenvolvimento de intervenções para a recuperação de repertórios prejudicados por transtornos neurocognitivos. Por outro lado, a complexidade da tarefa pode obscurecer aspectos mais básicos do desempenho dos idosos e que podem estar comprometidos em função do processo normal de envelhecimento, e, também, em função de comprometimento cognitivo em estado inicial. Nesse sentido, parece importante investigar adicionalmente o desempenho de idosos em tarefas de controle de estímulos mais simples. Estudos sobre o desempenho de idosos em tarefas de discriminação simples e em tarefas envolvendo relações de identidade podem auxiliar a elucidar eventuais dificuldades decorrentes do envelhecimento.

\section{Declaração de conflito de interesses}

A autora declara que não há conflito de interesses relativos à publicação deste artigo.

\section{Direitos Autorais}

Este é um artigo aberto e pode ser reproduzido livremente, distribuído, transmitido ou modificado, por qualquer pessoa desde que usado sem fins comerciais. $O$ trabalho é disponibilizado sob a licença Creative Commons 4.0 BY-NC.

(cc) BY-NC 


\section{Referências}

Aggio, N. M., Antoniazzi, L. C.K., \& Domenicone, C. (2008). Formação de classes de estímulos equivalentes em idosos com idade avançada. Revista Brasileira de Análise do Comportamento, 4(2), $219-230$. https://doi.org/10.18542/rebac.v4i2.852

Aggio, N. M., \& Domeniconi, C. (2012). Formação e manutenção de classes de estímulos equivalentes: Um estudo com participantes da terceira idade. Acta Comportamentalia, 20(1), 29-43.

Aggio, N. M., Ducatti, M., \& de Rose, J. C. (2018). Cognition and language in dementia patients: Contributions from behavior analysis. Behavioral Interventions, 33(3), 322-335. https://doi.org/10.1002/bin.1527

Ardila, A., Ostrosky-Solis, F., Rosselli, M., \& Gómez, C. (2000). Age-related cognitive decline during normal aging: The complex effect of education. Archives of Clinical Neuropsychology, 15(6), 495513.https://doi.org/10.1093/arclin/15.6.495

Arntzen, E., \& Holth, P. (1997). Probability of stimulus equivalence as a function of training design. The Psychological Record, 47, 309-320. https://doi.org/10.1007/BF03395227

Arntzen, E., \& Holth, P. (2000). Equivalence outcome in single subjects as a function of training structure. The Psychological Record, 50, 603-628. https://doi.org/10.1007/BF03395374

Cáceres Pachón, M. P., Gómez Bujedo, J., \& Lorca Marín, J. A. (2015). Diferencias en el aprendizaje y derivación de relaciones arbitrarias entre jóvenes y ancianos. Revista Argentina de Ciencias del Comportamiento, 7(3), 14-25.

de Rose, J. C., \& Bortoloti, R. (2007). A equivalência de estímulos como modelo do significado. Acta Comportamentalia, 15(3), 83-102.

de Rose, J. C. (1993). Classes de estímulos: implicações para uma análise comportamental da cognição. Psicologia: Teoria e Pesquisa, 9(2), 283-303.

Domeniconi, C., Zaine, I., Aggio, N. M., \& Benitez, P. (2010). Populações da terceira idade e equivalência de estímulos: Uma revisão bibliográfica. In: M. M. C. Hübner, M. R. Garcia, P. R. Abreu, E. N. P. de Cillo, \& P. B., Faleiros (Eds.), Sobre Comportamento e Cognição - vol. 26 (pp. 299-308). Santo André - SP: ESETec - Editores Associados.

Ducatti, M., \& Schmidt, A. (2016). Learning conditional relations in elderly people with and without neurocognitive disorders. Psychology \& Neuroscience, 9(2), 240-254. https://doi.org/10.1037/pne0000049

Fields, L., Arntzen, E., Nartey, R. K., \& Eilifsen, C. (2012). Effects of a meaningful, a discriminative, and a meaningless stimulus on equivalence class formation. Journal of the Experimental Analysis of Behavior, 97(2), $163-181$. https://doi.org/10.1901/jeab.2012.97-163

Folstein, M. F., Folstein, S. E., \& McHugh, P. R. (1975)." Mini-mental state. A practical method for grading the cognitive state of patients for the clinician. Journal of Psychiatric Research, 12, 189-198. https://doi.org/10.1016/00223956(75)90026-6

Gallagher, S. M., \& Keenan, M. (2009). Stimulus equivalence and the Mini Mental Status Examination in the elderly. European Journal of Behavior Analysis, 10(2), 159-165. https://doi.org/10.1080/15021149.2009.11434316

Goyos, C., Rossit, R. A. S., Elias, N. C., Escobal, G., \& Chereguini, P. (2009). Análise do comportamento e o estudo do envelhecimento humano: revisão dos estudos de aplicação. Revista Brasileira de Análise do Comportamento, 5(2), 1-20. http://dx.doi.org/10.18542/rebac.v5i2.927

Haydu, V. B., \& Morais, L. P. (2009). Formation, maintenance and reestablishment of equivalence relations with third age adults. Psicologia Argumento, 27(59), 323-336.

Holth, P., \& Arntzen, E. (2000). Reaction times and the emergence of class consistent responding: A case for precurrent responding?. The Psychological Record, 50(2), 305-337. https://doi.org/10.1007/BF03395358

Pérez-González, L. A., \& Moreno-Sierra, V. (1999). Equivalence class formation in elderly persons. Psicothema, 11(2), 325336.

Saunders, R. R., Chaney, L., \& Marquis, J. G. (2005). Equivalence class establishment with two-, three-, and four-choice matching to sample by senior citizens. The Psychological Record, 55, 539-559. https://doi.org/10.1007/BF03395526

Sidman, M. (1994). Equivalence relations and behavior: A research story. Boston: Authors Cooperative.

Sidman, M., \& Tailby, W. (1982). Conditional discrimination vs. matching to sample: An expansion of the testing paradigm. Journal of the Experimental Analysis of Behavior, 3711), 5-22. https://doi.org/10.1901/jeab.1982.37-5

Steingrimsdottir, H. S., \& Arntzen, E. (2011). Using conditional discrimination procedures to study remembering in an Alzheimer's patient. Behavioral Interventions, 26(3), 179-192. https://doi.org/10.1002/bin.334

Steingrimsdottir, H. S., \& Arntzen, E. (2014). Performance by older adults on identity and arbitrary matching-to-sample tasks. The Psychological Record, 64, 827-839. https://doi.org/10.1007/s40732-014-0053-8 
Trahan, M. A., Donaldson, J. M., McNabney, M. K., \& Kahng, S. (2014). Training and maintenance of a picture-based communication response in older adults with dementia. Journal of Applied Behavior Analysis, 47(2), 404-409. https://doi.org/10.1002/jaba.111

Trahan, M. A., Kahng, S., Fisher, A. B., \& Hausman, N. L. (2011). Behavior-analytic research on dementia in older adults. Journal of Applied Behavior Analysis, 44(3), 687-691. https://doi.org/10.1901/jaba.2011.44-687

Wilson, K. M., \& Milan, M. A. (1995). Age differences in the formation of equivalence classes. The Journals of Gerontology Series B: Psychological Sciences and Social Sciences, 50B(4), 212-218. https://doi.org/10.1093/geronb/50B.4.P212

Submetido em: $14 / 02 / 2020$

Aceito em: 08/12/2020 